\title{
Article
}

\section{Radiative transfer of synchrotron radiation through a compressed random magnetic field}

Cawthorne, Timothy Vernon

Available at http://clok.uclan.ac.uk/7530/

Cawthorne, Timothy Vernon (2013) Radiative transfer of synchrotron radiation through a compressed random magnetic field. The Astrophysical Journal, 771 (1). p. 60. ISSN 1538-4357

It is advisable to refer to the publisher's version if you intend to cite from the work. http://dx.doi.org/10.1088/0004-637x/771/1/60

For more information about UCLan's research in this area go to http://www.uclan.ac.uk/researchgroups/ and search for <name of research Group>.

For information about Research generally at UCLan please go to http://www.uclan.ac.uk/research/

All outputs in CLoK are protected by Intellectual Property Rights law, including Copyright law. Copyright, IPR and Moral Rights for the works on this site are retained by the individual authors and/or other copyright owners. Terms and conditions for use of this material are defined in the policies page.

\section{CLoK}

Central Lancashire online Knowledge www.clok.uclan.ac.uk

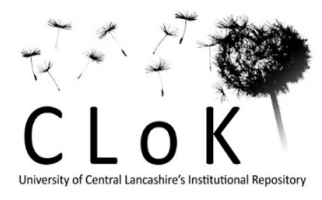




\title{
The radiative transfer of synchrotron radiation through a compressed random magnetic field.
}

\author{
T. V. Cawthorne \\ Jeremiah Horrocks Institute, University of Central Lancashire, Preston, Lancashire, PR1 \\ 2HE, U.K. \\ and \\ P. A. Hughes \\ Department of Astronomy, University of Michigan, Ann Arbor, MI 48109-1042, U.S.A.
}

\begin{abstract}
Magnetic field configurations resulting from the compression of a highly disordered magnetic field have found a wide range of applications in radio astronomy. For example, such structures may account for highly polarized emission near the edges of the lobes in radio galaxies (where the plane of $E$-field polarization is perpendicular to the edge of the lobe) and in the bright components in radio jets (where compression is thought to be due to shock waves and the plane of $E$-field polarization is often parallel to the jet). This paper shows that, provided Faraday rotation and circular polarization can be neglected, the radiative transfer equations for synchrotron radiation separate for this configuration, and the intensities and polarization values for sources that are uniform on large scales can be found straightforwardly in the case where opacity is significant. Although the emission and absorption coefficients must, in general, be obtained numerically, the process is much simpler than a full numerical solution to the transfer equations. Some illustrative results are given and an interesting effect, whereby the polarization increases while the magnetic field distribution becomes less strongly confined to the plane of compression, is discussed.
\end{abstract}


Subject headings: radio astronomy: radiation processes: synchrotron radiation, galaxies: active-galaxies:

\section{Introduction.}

Models for relativistic jet production in active galactic nuclei strongly favor ordered magnetic fields within thousands of gravitational radii of the central supermassive black hole (Agudo et al. 2012) even when complex flows and instability are admitted (McKinney et al. 2012; Porth 2013). Such fields are often assumed to persist to the parsec and even kiloparsec scale, and indeed might be required to explain observations as diverse as transverse gradients in Faraday rotation measure (Pudritz et al. 2012) and the extraordinary stability of flows such as that revealed by radio and X-ray observations of Pictor A (Wilson et al. 2001).

Nevertheless, compelling evidence exists that a substantial fraction of the magnetic field energy is in a random component, from the sub-parsec to kiloparsec scales. Following an analysis of cm-band single-dish data by Jones et al. (1985), which revealed a magnetic field structure capable of explaining the "rotator events" seen in time series data of Stokes parameters $Q$ and $U$, activity in a number of AGN has been successfully modeled by shocks that compress an initially tangled magnetic field, increasing the percentage polarization during outburst (Hughes et al. 1989, 1991). Such a model has recently been extended to incorporate oblique shocks (Hughes et al. 2011). Tangled magnetic fields carried through conical shock structures have been explored by Cawthorne (2006), and this picture has been successfully used to explain the characteristics of a stationary jet feature in 3C 120 (Agudo et al. 2012) and has been suggested as an explanation of multiwavelength variations of 3C 454.3 (Wehrle et al. 2012). On the larger (kiloparsec) scale, Laing \& Bridle (2002) have pioneered analysis of the magnetic field structure of jets, most recently concluding (Laing et al. 2006) that the jet in 3C 296 has a random but anisotropic magnetic field structure.

The spectral, spatial, and temporal behavior of the Stokes parameters $Q$ and $U$ provides a powerful diagnostic of the magnetic field structure, and thus indirectly, of the flow character in such jets, and the degree of linear polarization for a compressed, tangled magnetic field (due, for example, to a shock) was explored in the optically thin limit by Hughes et al. (1985). (An earlier paper, Laing (1980), also considered this kind of structure in the limit of an infinitely strong compression.) However, at least on the parsec and sub-parsec scale these flows exhibit opacity. Indeed, the "core" seen in low frequency $(\nu \leq 10 \mathrm{GHz})$ VLBI maps is widely interpreted as being the " $\tau=1$-surface": the location of the transition from optically thin to optically thick emission at the observing frequency of the map (Marscher 2006). This location within the jet has a special significance for jet studies, as it is by definition 
the surface from which propagating components first appear as distinct features on the map; there is compelling evidence that $\gamma$-ray flares arise close to the mm-wave core (Marscher et al. 2010), understanding the origin of which requires knowledge of the flow conditions there. At these higher frequencies, the interpretation of the core as the $\tau=1$-surface is certainly complicated by the presence of stationary features (possibly recollimation shocks) which, even if responsible for the core in some sources, must lie close to regions of significant opacity (Marscher 2006). It would therefore be of great value to have a description of the polarized emission from compressed, tangled magnetic fields in the presence of opacity.

\section{Propagation of synchrotron radiation through a compressed random field.}

This section demonstrates that the radiative transfer equations for the propagation of synchrotron radiation through a compressed random field separate, provided circular polarization and Faraday rotation can be neglected. The resulting absorption and emission coefficients are obtained in Appendix A. The approach follows those of Appendix A in Hughes, Aller \& Aller (1985) and Chapter 3 from Pacholczyk (1970). In order to obtain consistency between these two works, the coordinate system used in Hughes, Aller \& Aller have been relabelled as follows.

Before compression, the direction of the magnetic field is defined by reference to the $\left(a^{\prime}, b^{\prime}, c^{\prime}\right)$ coordinate system as shown in Fig. 1 (left panel). The polar angle $\theta$ separates the $a^{\prime}$ axis and the direction of the local magnetic field, and the azimuthal angle $\phi$ separates the $c^{\prime}$ axis and the projection of the field onto the $b^{\prime}, c^{\prime}$ plane. In this system, $B_{a^{\prime}}=B_{0} \cos \theta$, $B_{b^{\prime}}=B_{0} \sin \theta \sin \phi$ and $B_{c^{\prime}}=B_{0} \sin \theta \cos \phi$. After compression, such that unit length parallel to the $a^{\prime}$ axis is reduced to length $K$, the requirement that magnetic flux is conserved yields a magnetic field with components $B_{a^{\prime}}=B_{0} \cos \theta, B_{b^{\prime}}=B_{0} \sin \theta \sin \phi / K$ and $B_{c^{\prime}}=$ $B_{0} \sin \theta \cos \phi / K$. A rotation of the coordinate system through angle $\delta$ about the $b^{\prime}$ axis gives the $a, b, c$ coordinate system (chosen so that the observer lies on the $-c$ axes) in terms of which the local magnetic field is

$$
\begin{aligned}
B_{a} & =B_{0}(\cos \theta \cos \delta+\sin \theta \cos \phi \sin \delta / K) \\
B_{b} & =B_{0} \sin \theta \sin \phi / K \\
B_{c} & =B_{0}(\sin \theta \cos \phi \cos \delta / K-\cos \theta \sin \delta)
\end{aligned}
$$

These results can be obtained from Hughes, Aller \& Aller (1985) by making the substitutions $\left(x \rightarrow-c^{\prime}, y \rightarrow b^{\prime}, z \rightarrow a^{\prime}, x^{\prime} \rightarrow-c, y^{\prime} \rightarrow b, z^{\prime} \rightarrow a\right.$, and $\left.\epsilon \rightarrow-\delta\right)$.

Following Pacholczyk (1970) Equation 3.66 and assuming that the circular polarization 

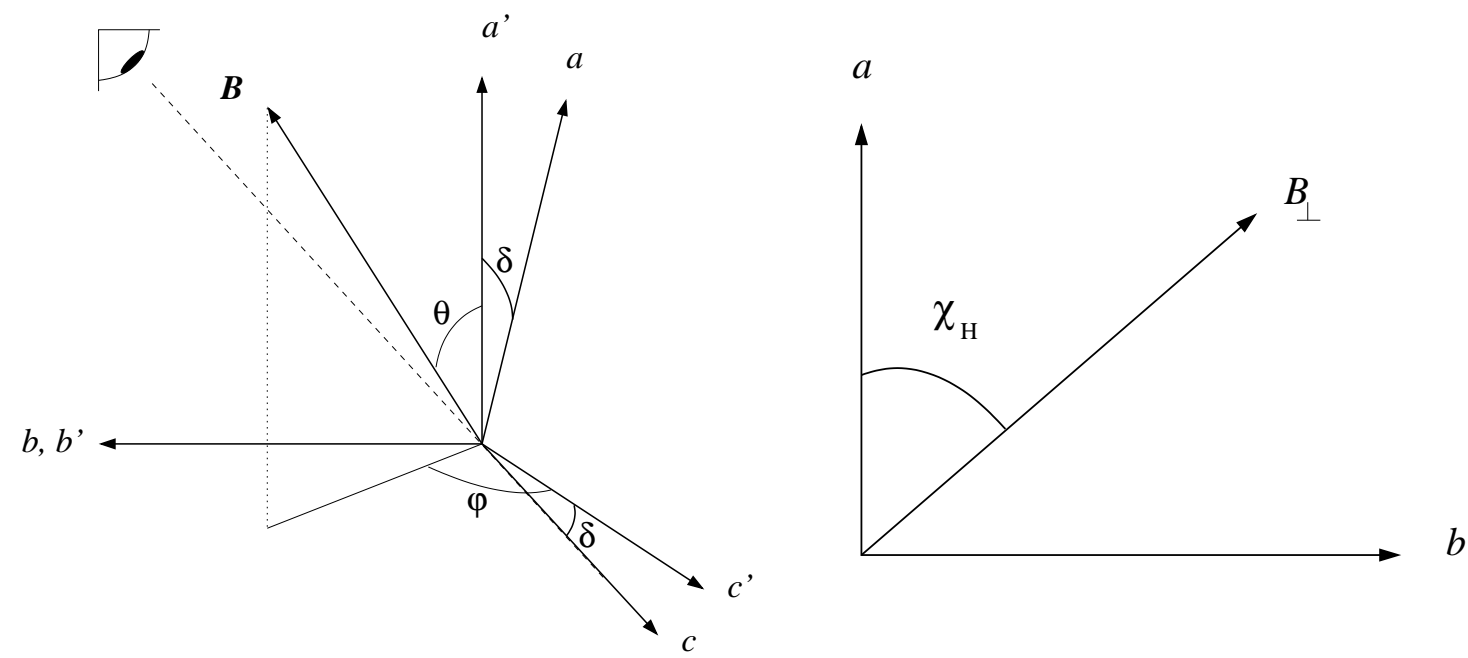

Fig. 1.- Left diagram: This figure illustrates the coordinate systems used in this paper. Both the $a$ and $a^{\prime}$ axes and the $c$ and $c^{\prime}$ axes are inclined at angle $\delta$. The $b$ and $b^{\prime}$ axes are coincident. The magnetic field is defined with respect to the $\left(a^{\prime}, b^{\prime}, c^{\prime}\right)$ coordinate system. Plasma with disordered magnetic field is compressed along the direction parallel to the $a^{\prime}$ axis. Radiation is observed propagating along the $-c$ axis. Right diagram: This figure illustrates the sky plane, with the $c$ axis pointing away from the observer. $\chi_{H}$ is the angle between the $a$ axis and the projection of the magnetic field onto the sky plane. 
and Faraday rotation are negligible, the radiative transfer equations are written in terms of $I^{(a)}$ and $I^{(b)}$, the intensities measured by dipoles aligned with the $a$ and $b$ axes, respectively, and the Stokes parameter $U^{(a b)}$ :

$$
\begin{aligned}
\frac{d I^{(a)}}{d s} & =I^{(a)}\left[-\kappa^{(1)} \sin ^{4} \chi_{H}-\kappa^{(2)} \cos ^{4} \chi_{H}-\frac{1}{2} \kappa \sin ^{2} 2 \chi_{H}\right] \\
& +U^{(a b)}\left[\frac{1}{4}\left(\kappa^{(1)}-\kappa^{(2)}\right) \sin 2 \chi_{H}\right]+\epsilon^{(1)} \sin ^{2} \chi_{H}+\epsilon^{(2)} \cos ^{2} \chi_{H} \\
\frac{d I^{(b)}}{d s} & =I^{(b)}\left[-\kappa^{(1)} \cos ^{4} \chi_{H}-\kappa^{(2)} \sin ^{4} \chi_{H}-\frac{1}{2} \kappa \sin ^{2} 2 \chi_{H}\right] \\
& +U^{(a b)}\left[\frac{1}{4}\left(\kappa^{(1)}-\kappa^{(2)}\right) \sin 2 \chi_{H}\right]+\epsilon^{(1)} \cos ^{2} \chi_{H}+\epsilon^{(2)} \sin ^{2} \chi_{H} \\
\frac{d U^{(a b)}}{d s} & =\left(I^{(a)}+I^{(b)}\right) \frac{1}{2}\left(\kappa^{(1)}-\kappa^{(2)}\right) \sin 2 \chi_{H}-\kappa U^{(a b)}-\left(\epsilon^{(1)}-\epsilon^{(2)}\right) \sin 2 \chi_{H}
\end{aligned}
$$

Here, $\chi_{H}$ is the angle between the $a$ axis and the projection of the magnetic field onto the plane of the sky, as shown in Fig. 1 (right diagram). $\kappa^{(1)}$ and $\kappa^{(2)}$ are, respectively, the absorption coefficients for planes of (electric field) polarization perpendicular and parallel to the projected magnetic field. Likewise, $\epsilon^{(1)}$ and $\epsilon^{(2)}$ are, respectively, the emission coefficients for planes of (electric field) polarization perpendicular and parallel to the projected magnetic field. The polarization-averaged absorption coefficient is defined by

$$
\kappa=\left(\kappa^{(1)}+\kappa^{(2)}\right) / 2
$$

For a power-law distribution of radiating electrons such that the density of electrons in the energy interval $d E$ is $\mathcal{N}(E) d E=\mathcal{N}_{0} E^{-\gamma} d E$, the emission and absorption coefficients for a region with uniform field are given by Pacholczyk (1970) as

$$
\begin{aligned}
\epsilon^{(1),(2)} & =C \mathcal{N}_{0} B_{\perp}^{(1+\gamma) / 2} \nu^{(1-\gamma) / 2}\left[1 \pm \frac{\gamma+1}{\gamma+7 / 3}\right] \\
\kappa^{(1),(2)} & =D \mathcal{N}_{0} B_{\perp}^{(2+\gamma) / 2} \nu^{-(4+\gamma) / 2}\left[1 \pm \frac{\gamma+2}{\gamma+10 / 3}\right]
\end{aligned}
$$

where the constants $C$ and $D$ are given in Appendix B. Inside the square brackets, the plus sign refers to polarization (1) and the minus sign to polarization (2).

\subsection{Separation of the transfer equations.}

Equations 4 to 6 contain a term describing the contribution to $I^{(a),(b)}$ and $U^{(a b)}$ due to polarized absorption, which depends on $\left(\kappa^{(1)}-\kappa^{(2)}\right) \sin 2 \chi_{H}$. For the power-law distribution 
of particles considered here it is always true that $\kappa^{(1)}>\kappa^{(2)}$, Thus in the (12) frame, these contributions are always in the same sense. For a uniform magnetic field this term is zero because polarized absorption orthogonal to the field does not contribute to the mode parallel to the field, and vice versa. If we consider a partially compressed random magnetic field as equivalent to the sum of a uniform component orthogonal to the sense of compression, plus a superposed random distribution of field elements, the latter will not reintroduce contributions from these difference terms, as their random distribution guarantees that they do not modify the polarized component of the radiation. Equivalently, while a compressed magnetic field exhibits a preferred sense - the plane of compression - and the projected magnetic field elements will be distributed with a narrow dispersion in $\chi_{H}$ about the projection of this direction on the plane of the sky, on average there will be as many elements with $\chi_{H}>0$ as there are with $\chi_{H}<0$, with no net effect upon the radiation field. A more formal demonstration of this result follows.

From Equations 4 and 5,

$$
\begin{aligned}
\kappa^{(1)}-\kappa^{(2)} & =D \mathcal{N}_{0} B_{\perp}^{(2+\gamma) / 2} \nu^{-(4+\gamma) / 2}\left[\frac{2(\gamma+2)}{\gamma+10 / 3}\right] \\
& =D \mathcal{N}_{0}\left(B_{a}^{2}+B_{b}^{2}\right)^{(2+\gamma) / 4} \nu^{-(4+\gamma) / 2}\left[\frac{2(\gamma+2)}{\gamma+10 / 3}\right]
\end{aligned}
$$

From Fig. 1 (right diagram),

$$
\begin{aligned}
\sin \chi_{H} & =\frac{B_{b}}{\left(B_{a}^{2}+B_{b}^{2}\right)^{1 / 2}} \\
\cos \chi_{H} & =\frac{B_{a}}{\left(B_{a}^{2}+B_{b}^{2}\right)^{1 / 2}}
\end{aligned}
$$

and so

$$
\sin 2 \chi_{H}=2 B_{a} B_{b} /\left(B_{a}^{2}+B_{b}^{2}\right)
$$

so that $\left(\kappa^{(1)}-\kappa^{(2)}\right) \sin 2 \chi_{H} \propto B_{a} B_{b}\left(B_{a}^{2}+B_{b}^{2}\right)^{(\gamma-2) / 4}$. Averaging this expression over all $\theta$ and $\phi$ gives

$$
<\left(\kappa^{(1)}-\kappa^{(2)}\right) \sin 2 \chi_{H}>\propto \frac{1}{4 \pi} \int_{-\pi}^{\pi} \int_{0}^{\pi} B_{a} B_{b}\left(B_{a}^{2}+B_{b}^{2}\right)^{(\gamma-2) / 4} \sin \theta d \theta d \phi
$$

From Equations 1 to 3, it is clear that $B_{a}$ is an even function of $\phi$ while $B_{b}$ is an odd function of $\phi$, so that the integrand in Equation 14 is an odd function of $\phi$. Integrating with respect to $\phi$ from $-\pi$ to $\pi$ therefore yields the result zero. Therefore

$$
<\left(\kappa^{(1)}-\kappa^{(2)}\right) \sin 2 \chi_{H}>=0
$$


Hence, $d I^{(a)} / d s$ depends only on $I^{(a)}, d I^{(b)} / d s$ depends only on $I^{(b)}$ and $d U^{(a b)} / d s$ depends only on $U^{(a b)}$. In this case, the equations separate and have straightforward solutions.

Very similar similar arguments apply to the term $\left(\epsilon^{(1)}-\epsilon^{(2)}\right) \sin 2 \chi$ in Equation 6, which describes the contribution of polarized emission to $U^{(a b)}$, so that $<\left(\epsilon^{(1)}-\epsilon^{(2)}\right) \sin 2 \chi_{H}>=0$.

Assuming that the magnetic field is disordered on scales small compared those over which the radiation field changes significantly, the emission and absorption coefficients can be averaged over initial magnetic field direction and the radiative transfer Equations 4 to 6 thus simplify to

$$
\begin{aligned}
\frac{d I^{(a)}}{d s} & =-<\kappa^{(a)}>I^{(a)}+<\epsilon^{(a)}> \\
\frac{d I^{(b)}}{d s} & =-<\kappa^{(b)}>I^{(b)}+<\epsilon^{(b)}> \\
\frac{d U^{(a b)}}{d s} & =-<\kappa>U^{(a b)}
\end{aligned}
$$

for which, in a uniform source, the following solutions can be obtained straightforwardly:

$$
\begin{aligned}
I^{(a)}(s) & =\frac{<\epsilon^{(a)}>}{<\kappa^{(a)}>}\left(1-\exp \left(-<\kappa^{(a)}>s\right)\right)+I^{(a)}(s=0) \exp \left(-<\kappa^{(a)}>s\right) \\
I^{(b)}(s) & =\frac{<\epsilon^{(b)}>}{<\kappa^{(b)}>}\left(1-\exp \left(-<\kappa^{(b)}>s\right)\right)+I^{(b)}(s=0) \exp \left(-<\kappa^{(b)}>s\right) \\
U^{(a b)}(s) & =U^{(a b)}(s=0) \exp (-<\kappa>s)
\end{aligned}
$$

where $I^{(a)}(s=0), I_{b}(s=0)$ and $U^{(a b)}(s=0)$, are the values incident upon the source, $s$ is the path length through the source, and

$$
\begin{aligned}
\epsilon^{(a)} & =\epsilon^{(1)} \sin ^{2} \chi_{H}+\epsilon^{(2)} \cos ^{2} \chi_{H} \\
\epsilon^{(b)} & =\epsilon^{(1)} \cos ^{2} \chi_{H}+\epsilon^{(2)} \sin ^{2} \chi_{H} \\
\kappa^{(a)} & =\kappa^{(1)} \sin ^{4} \chi_{H}+\kappa^{(2)} \cos ^{4} \chi_{H}+\frac{1}{2} \kappa \sin ^{2} 2 \chi_{H} \\
\kappa^{(b)} & =\kappa^{(1)} \cos ^{4} \chi_{H}+\kappa^{(2)} \sin ^{4} \chi_{H}+\frac{1}{2} \kappa \sin ^{2} 2 \chi_{H}
\end{aligned}
$$

\section{Results.}

Appendix A shows how the emission coefficients $\left\langle\epsilon^{(a),(b)}>\right.$ and absorption coefficients $<\kappa^{(a),(b)}>$ can be expressed in terms of the function $F_{\gamma}^{(a),(b)}$ (Equations A5 to A7 and 
Equation A11). The integrals in these expressions are not, in general, analytically tractable, but since $F_{\gamma=3}^{(a),(b)}$ has a simple solution (Equations A14, A15 and A16), simple formulae exist for the emission coefficients when $\gamma=3$ and for the absorption coefficients when $\gamma=2$. A rough analytical approximation to $F_{\gamma=2}^{(a),(b)}$ is given by Equations A17 and $A 18$ and correction factors are plotted in Fig. 5. These allow computation of intensities and polarization in the case $\gamma=2$ without resort to a computer. Expressions for the constants $C, D$ and $\mu$ are given in Appendix B, and their values are given in Table 1 for some values of $\gamma$ in the range of greatest interest.

Results illustrating how the emergent polarization varies with frequency $\nu$ and line of sight angle $\delta$ are presented below. The integrals were performed numerically using Simpson's rule with 50 evaluations per integral. Comparison between results that can be obtained analytically and the corresponding values obtained numerically suggests that the latter are accurate four significant figures at least.

\subsection{Polarization as a function of frequency.}

If the source is uniform on scales over which the intensity changes significantly, then the solutions given by Equations 19, 20 and 21 apply. It is convenient to define a characteristic frequency, $\nu_{0}$, at which the polarization averaged opacity is unity, i.e.,

$$
<\kappa>L=\left(<\kappa^{(1)}>+<\kappa^{(2)}>\right) L / 2=1
$$

(Note that $\nu_{0}$ will be a function of $K, \delta$ and $\gamma$.) Then, from Equations A11 to A12, the opacities in polarizations $a$ and $b$ are

$$
\tau^{(a),(b)}=\left\langle\kappa^{(a),(b)}>L=\left(\frac{\nu}{\nu_{0}}\right)^{-(\gamma+4) / 2} \frac{F_{(\gamma+1)}^{(a),(b)}(\delta, K)}{H_{\gamma}(\delta, K)}\right.
$$

The intensities are then given by Equations 19, 20 and B9

$$
I^{(a),(b)}=\frac{\mu m \nu^{5 / 2}}{\nu_{L}^{1 / 2}} \frac{F_{\gamma}^{(a),(b)}}{F_{\gamma+1}^{(a),(b)}}\left(1-e^{-\tau^{(a),(b)}}\right)
$$

and the degree of polarization is then

$$
\Pi=\frac{I^{(a)}-I^{(b)}}{I^{(a)}+I^{(b)}}
$$

The spectral variation of the degree of polarization is illustrated in Fig. 2 for three values of $\delta$, two values of $\gamma$, and $K=0.2$. The figure illustrates the transition from optically thin 


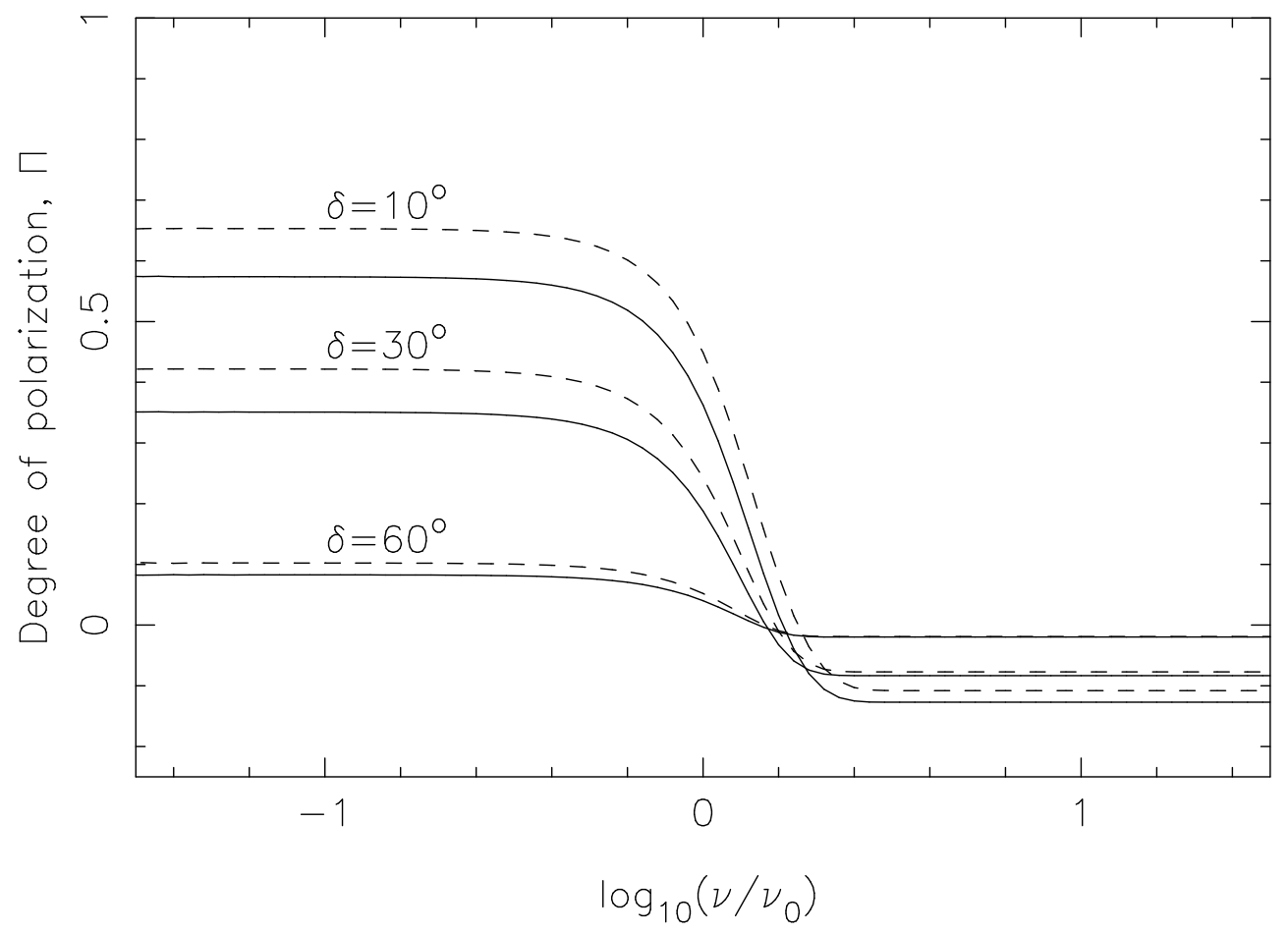

Fig. 2. - The degree of polarization as a function of frequency for three values of the inclination angle, $\delta$. The continuous lines are for $\gamma=2$, the dashed lines for $\gamma=3$. The compression factor is $K=0.2$. 
emission, where the polarization fraction is generally high and the ( $E$ field) polarization direction is parallel to the $a$ axis $(\Pi>0)$, to optically thick emission, where the polarization is generally lower, and the polarization direction is parallel to the $b$ axis $(\Pi<0)$. The polarization decreases as $\delta$, the angle of inclination between the line of sight and the plane of compression, increases, and the disordered component of the magnetic field becomes more apparent.

In the optically thin (high frequency) limit, the degree of polarization is

$$
\Pi_{\text {thin }}=\frac{F_{\gamma}^{a}-F_{\gamma}^{(b)}}{F_{\gamma}^{(a)}+F_{\gamma}^{(b)}}
$$

while in the optically thick (low frequency) limit, the degree of polarization is

$$
\Pi_{\text {thick }}=\frac{\left(F_{\gamma}^{(a)} / F_{\gamma+1}^{(a)}\right)-\left(F_{\gamma}^{(b)} / F_{\gamma+1}^{(b)}\right)}{\left(F_{\gamma}^{(a)} / F_{\gamma+1}^{(a)}\right)+\left(F_{\gamma}^{(b)} / F_{\gamma+1}^{(b)}\right)}
$$

These values are plotted as a function of compression factor $K$, for various values of the inclination angle $\delta$, in Fig 3. As expected, in the optically thin limit, the degree of polarization decreases monotonically with increasing $K$, and with increasing $\delta$. The value of $\Pi$ in the optically thick limit generally decreases in magnitude as $K$ increases, though for $\delta$ less than about $10^{\circ}$, the value of $\Pi_{\text {thick }}$ has a turning point at about $K=0.2$. It is, at first sight, surprising that, as $K$ increases from zero and the field becomes more isotropic (or less strongly confined to the plane of compression), the degree of polarization actually increases. This occurs because, although both emission and absorption coefficients for the two polarizations become closer, as clearly they should, the values of $\epsilon / \kappa$ for the two polarizations initially diverge. The reason is that, while $K$ is very small and increasing, both $\epsilon^{(a)} / \epsilon^{(b)}$ and $\kappa^{(a)} / \kappa^{(b)}$ decrease, but the ratio of the $\epsilon$ values decreases more strongly than that of the $\kappa$ values. This occurs because the contribution to the coefficients from the component of field perpendicular to the plane of compression (which, in relative terms, is increasing) is greater for the emission coefficients than the absorption coefficients, because the latter depend more sensitively on magnetic field. This subtle effect can be more easily understood with reference to a similar but simpler magnetic field geometry, as shown in Appendix C.

\subsection{Polarization as a function of inclination.}

The dependence of the degree of polarization upon $\delta$, the angle of inclination between the line of sight and plane of compression, is described below. If the emitting plasma is 

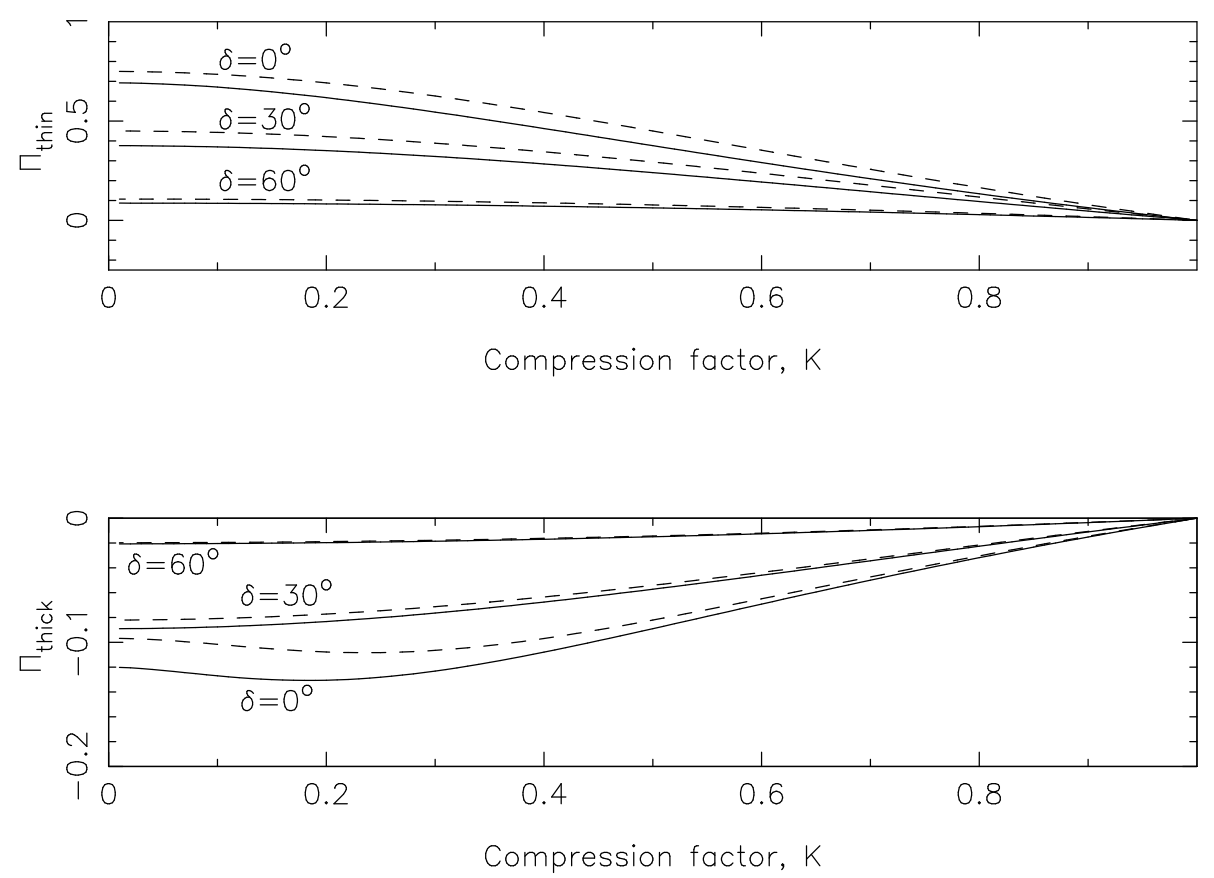

Fig. 3.- The degrees of polarization in the optically thin limit (above) and the optically thick limit (below) are plotted as a function of $K$ for the values of $\delta$ shown. The continuous lines show results for $\gamma=2$, the dashed lines for $\gamma=3$. 
confined between two planes, each parallel to the plane of compression and separated by a distance $w$, then the path length through the plasma is $L=w / \sin \delta$. The opacity is characterised by the value $\tau_{0}=\left\langle\kappa>\left(\delta=90^{\circ}, K, \gamma\right) w\right.$, the polarization averaged opacity when the line of sight is perpendicular to the plane of compression. The value of $\tau_{0}$ is given by

$$
\tau_{0}=D \mathcal{N}_{0}(K) B_{0}^{(2+\gamma) / 2} \nu^{-(4+\gamma) / 2} H_{\gamma}\left(\delta=90^{\circ}, K\right) w
$$

Then, if $\delta$ is varied while $K, \gamma$ and $\nu$ remain fixed,

$$
\tau^{(a),(b)}=\tau_{0} \frac{F_{\gamma+1}^{(a),(b)}(\delta, K)}{H_{\gamma}\left(\delta=90^{\circ}, K\right) \sin \delta}
$$

The intensities $I^{(a)}$ and $I^{(b)}$ and the degree of polarization are then given by Equations 29 and 30. The results are shown in Fig. 4, in which $\Pi$, the degree of polarization, is plotted against $\delta$, for a compression factor $K=0.2$, and values of $\tau_{0}=0.25,1.0,4.0$. The results show that, as $\delta$ decreases from $90^{\circ}$, the polarization first rises as the partial order of the magnetic field becomes more apparent, but then starts to fall, as opacity begins to take effect. As $\delta$ decreases further, $\Pi$ changes from positive to negative in value (i.e. the polarization angle changes by $90^{\circ}$ ), and the degree of polarization approaches the optically thick limit shown in Fig 3. As $\tau_{0}$ increases in value, the maximum (positive) value of $\Pi$ decreases and the frequency at which $\Pi$ changes from positive to negative increases.

\section{Summary of results.}

The radiative transfer equations for synchrotron radiation have been shown to separate for the case of propagation through a compressed, random magnetic field, provided Faraday

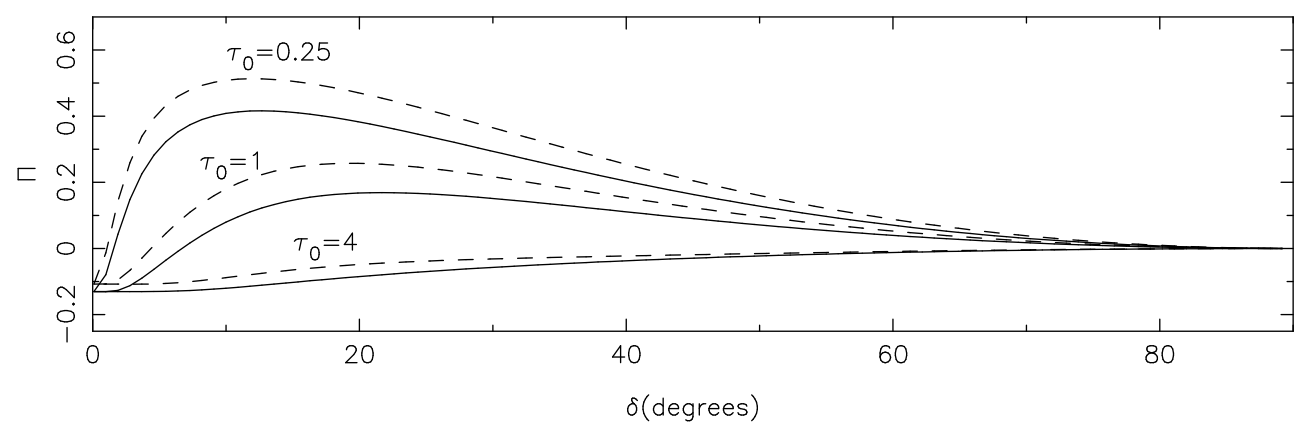

Fig. 4. - The degree of polarization is plotted as a function of $\delta$ for $K=0.2$ and the values of $\tau_{0}$ shown. Continuous lines show results for $\gamma=2$, dashed lines for $\gamma=3$. 
rotation and circular polarization can be neglected. Although, in general, the emission and absorption coefficients must be computed numerically, this is still much simpler than a full numerical solution of the coupled equations. Expressions for the emission and absorption coefficients are given in Appendix A. Exact analytical expressions result only for the emission coefficients when (energy index) $\gamma=3$, and for the absorption coefficients when $\gamma=2$. A rough approximation, together with a plot of correction factors, is given to allow calculation the emission coefficient for $\gamma=2$. This allows the solution to be found for a source that is uniform on large-scales, for $\gamma=2$, without resort to a computer.

Some illustrative results are presented, showing the variation of polarization with frequency, and with inclination of the plane of compression to the line of sight. The optically thin and thick limits to fractional polarization are plotted against compression factor, $K$, for various inclination angles. When the inclination angle $\delta<10^{\circ}$, the optically thick limit reveals an unusual trend in which, for very small $K$, the polarization increases as $K$ increases, i.e., as the magnetic field becomes less strongly confined to the plane of compression. This effect is discussed in the context of a simpler magnetic field model in Appendix C.

\section{Acknowledgements}

TVC thanks the director of the Jeremiah Horrocks Institute at the University of Central Lancashire for a sabbatical semester, during which most of the present work was undertaken. He also thanks Dr. J.-L. Gomez and the Instituto di Astrofisica de Aldalucia in Granada for their generous hospitality during a part of the sabbatical. This work arose from discussions with Dr. Gomez during that visit. PAH was partially supported by NASA Fermi GI grant NNX11AO13G during this work.

\section{A. Computation of the emission and absorption coefficients.}

Following the approach of Hughes, Aller \& Aller (1985), it is convenient to define the functions $M$ and $N$ such that

$$
\begin{aligned}
M(\theta, \phi) & =\left(B_{a} / B_{0}\right)^{2}=(\cos \theta \cos \delta+\sin \theta \cos \phi \sin \delta / K)^{2} \\
N(\theta, \phi) & =\left(B_{b} / B_{0}\right)^{2}=(\sin \theta \sin \phi / K)^{2}
\end{aligned}
$$

Furthermore, for a 1-D adiabatic compression, the particle density per unit energy, $\mathcal{N}_{0}=$ $\mathcal{N}_{0}(K) \propto K^{-(\gamma+2) / 3}$ (e.g., Hughes, Aller, \& Aller (1989)). Then, from Equation 22, the 
emission coefficient $\epsilon^{(a)}$ becomes

$$
\begin{aligned}
\epsilon^{(a)} & =\epsilon^{(1)} \sin ^{2} \chi_{H}+\epsilon^{(2)} \cos ^{2} \chi_{H} \\
& =C \mathcal{N}_{0}(K)\left(B_{a}{ }^{2}+B_{b}{ }^{2}\right)^{(1+\gamma) / 4} \nu^{(1-\gamma) / 2}\left(\frac{2 \gamma+10 / 3}{\gamma+7 / 3} \frac{B_{b}^{2}}{B_{a}^{2}+B_{b}^{2}}+\frac{4 / 3}{\gamma+7 / 3} \frac{B_{a}^{2}}{B_{a}^{2}+B_{b}^{2}}\right) \\
& =C \mathcal{N}_{0}(K) B_{0}^{(\gamma+1) / 2} \nu^{(1-\gamma) / 2}(M+N)^{(\gamma-3) / 4}\left(\frac{(2 \gamma+10 / 3) N+(4 / 3) M}{\gamma+7 / 3}\right) \\
& =C \mathcal{N}_{0}(K) B_{0}^{(\gamma+1) / 2} \nu^{(1-\gamma) / 2}\left(\frac{\frac{4}{3}(M+N)^{(\gamma+1) / 4}+2(\gamma+1) N(M+N)^{(\gamma-3) / 4}}{\gamma+7 / 3}\right)
\end{aligned}
$$

Similarly, from Equation 23

$$
\begin{aligned}
\epsilon^{(b)} & =\epsilon^{(1)} \cos ^{2} \chi_{H}+\epsilon^{(2)} \sin ^{2} \chi_{H} \\
& =C \mathcal{N}_{0}(K) B_{0}^{(\gamma+1) / 2} \nu^{(1-\gamma) / 2}\left(\frac{\frac{4}{3}(M+N)^{(\gamma+1) / 4}+2(\gamma+1) M(M+N)^{(\gamma-3) / 4}}{\gamma+7 / 3}\right)(
\end{aligned}
$$

Averaging over the initial magnetic field direction, the emission coefficients become

$$
<\epsilon^{(a),(b)}>=C \mathcal{N}_{0}(K) B_{0}^{(\gamma+1) / 2} \nu^{(1-\gamma) / 2} F_{\gamma}^{(a),(b)}(\delta, K)
$$

where

$$
F_{\gamma}^{(a)}(\delta, K)=\frac{1}{4 \pi} \int_{-\pi}^{\pi} \int_{0}^{\pi} \frac{\frac{4}{3}(M+N)^{(\gamma+1) / 4}+2(\gamma+1) N(M+N)^{(\gamma-3) / 4}}{(\gamma+7 / 3)} \sin \theta d \theta d \phi
$$

and

$$
F_{\gamma}^{(b)}(\delta, K)=\frac{1}{4 \pi} \int_{-\pi}^{\pi} \int_{0}^{\pi} \frac{\frac{4}{3}(M+N)^{(\gamma+1) / 4}+2(\gamma+1) M(M+N)^{(\gamma-3) / 4}}{(\gamma+7 / 3)} \sin \theta d \theta d \phi
$$

The absorption coefficients are given by Equations 9,24 and 25. It is convenient to express $\kappa^{(a)}$ and $\kappa^{(b)}$ in terms of the polarization averaged absorption coefficient, $\kappa$ (Equation 7 ), so that

$$
\begin{aligned}
\kappa^{(a)} & =\kappa\left(\frac{2 \gamma+16 / 3}{\gamma+10 / 3} \sin ^{4} \chi_{H}+\frac{4 / 3}{\gamma+10 / 3} \cos ^{4} \chi_{H}+\frac{1}{2} \sin ^{2} 2 \chi_{H}\right) \\
& =\kappa\left(\frac{4 / 3+2(\gamma+2) \sin ^{2} \chi_{H}}{\gamma+10 / 3}\right) \\
\kappa^{(b)} & =\kappa\left(\frac{2 \gamma+16 / 3}{\gamma+10 / 3} \cos ^{4} \chi_{H}+\frac{4 / 3}{\gamma+10 / 3} \sin ^{4} \chi_{H}+\frac{1}{2} \sin ^{2} 2 \chi_{H}\right) \\
& =\kappa\left(\frac{4 / 3+2(\gamma+2) \cos ^{2} \chi_{H}}{\gamma+10 / 3}\right),
\end{aligned}
$$


and

$$
\kappa=D \mathcal{N}_{0}(K) B_{0}^{(2+\gamma) / 2} \nu^{-(4+\gamma) / 2}(M+N)^{(2+\gamma) / 4}
$$

The values of $\kappa^{(a)}$ and $\kappa^{(b)}$ averaged over the initial magnetic field direction are thus

$$
\begin{aligned}
<\kappa^{(a),(b)}> & =D \mathcal{N}_{0}(K) B_{0}^{(2+\gamma) / 2} \nu^{-(4+\gamma) / 2} F_{\gamma+1}^{(a),(b)}(\delta, K) \\
<\kappa> & =D \mathcal{N}_{0}(K) B_{0}^{(2+\gamma) / 2} \nu^{-(4+\gamma) / 2} H_{\gamma}(\delta, K)
\end{aligned}
$$

where

$$
H_{\gamma}(\delta, K)=\frac{\int_{-\pi}^{\pi} \int_{0}^{\pi}(M+N)^{(2+\gamma) / 4} \sin \theta d \theta d \phi}{4 \pi}
$$

Unfortunately, the integrals appearing above are not, in general, analytically tractable. However, it is straightforward to evaluate $F_{\gamma}^{(a),(b)}$ if $\gamma=3$. The results are

$$
\begin{aligned}
F_{\gamma=3}^{(a)}(\delta, K) & =\frac{7+K^{2}+\sin ^{2} \delta\left(1-K^{2}\right)}{12 K^{2}} \\
F_{\gamma=3}^{(b)}(\delta, K) & =\frac{7 K^{2}+1+7 \sin ^{2} \delta\left(1-K^{2}\right)}{12 K^{2}} \\
H_{\gamma=3}(\delta, K) & =\frac{1+K^{2}+\sin ^{2} \delta\left(1-K^{2}\right)}{3 K^{2}}
\end{aligned}
$$

These results allow analytical calculation of the emission coefficients if $\gamma=3$ or the absorption coefficients if $\gamma=2$.

In an attempt to provide a means of calculating intensities without the aid of a computer various approximate solutions to the integrals for the $F$ and $H$ functions were attempted. The more sophisticated approaches, such as rational function approximations, were not successful. The best results overall were obtained by setting $\gamma=2$ and making the rather crude approximation that $\left(K^{2}(M+N)\right)^{1 / 4} \simeq 1$ in Equations $\mathrm{A} 6$ and A7. In that case,

$$
\begin{aligned}
& F_{\gamma=2}^{(a)}(\delta, K) \simeq f^{(a)}(\delta, K)=\frac{2}{39 K^{(3 / 2)}}\left(11+2 K^{2}+2 \sin ^{2} \delta\left(1-K^{2}\right)\right) \\
& F_{\gamma=2}^{(b)}(\delta, K) \simeq f^{(b)}(\delta, K)=\frac{2}{39 K^{(3 / 2)}}\left(2+11 K^{2}+11 \sin ^{2} \delta\left(1-K^{2}\right)\right)
\end{aligned}
$$

The approximation for $F^{(a)}$ are accurate to within $20 \%$, while that for $F^{(b)}$ is accurate to within $30 \%$. While this is not very helpful by itself, suitable correction factors, $T^{(a),(b)}$, where

$$
F_{\gamma=2}^{(a),(b)}(\delta, K)=f^{(a),(b)}(\delta, K) \times T^{(a),(b)}(\delta, K)
$$

are plotted in Fig. 5. In combination with Equations A14 and A15, these results allow intensities and degrees of polarization to be determined for energy index $\gamma=2$, without resort to a computer. 


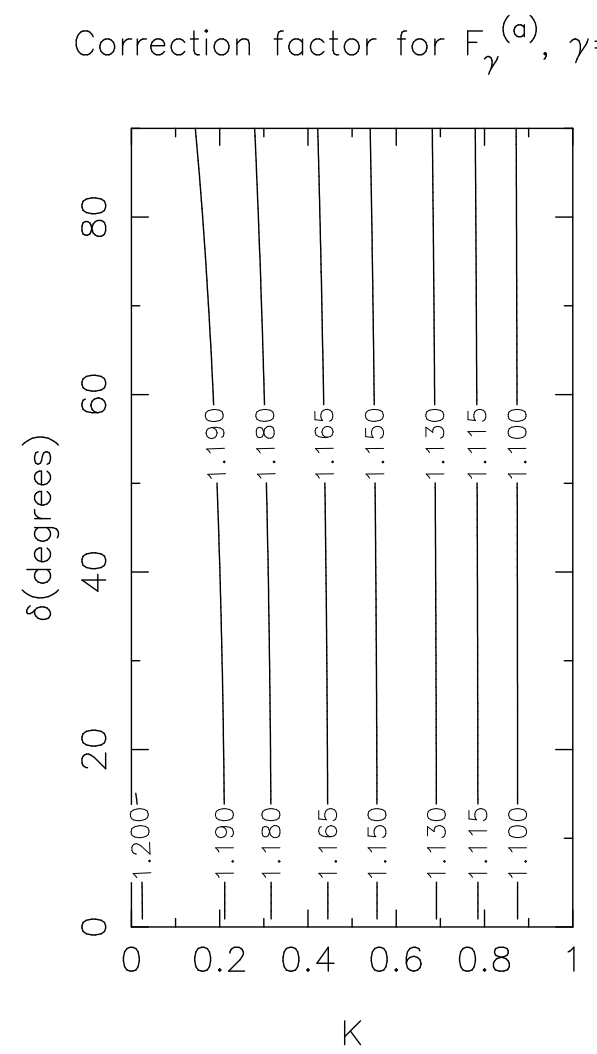

Correction factor for $F_{\gamma}^{(b)}, \gamma=2$

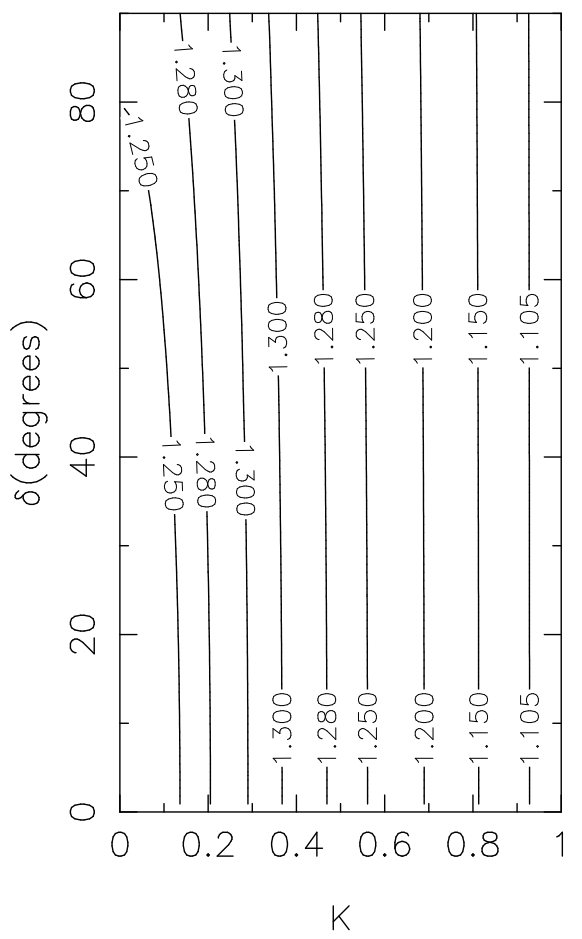

Fig. 5.- This figure shows Factors for the correction of the approximate forms for $F^{(a)}$ and $F^{(b)}$ given in Equations A17 and A18. 


\section{B. Constants.}

Pacholczyk's treatment of synchrotron radiation in a uniform magnetic field involves a large number of physical constants which are helpful in the derivations he performs. However, here, it is the constants of proportionality, $C$ and $D$, (which are actually functions of $\gamma$ ) that are of greatest interest and expressions for them are given here. Additionally, the formulae are converted from the obsolete CGS system to SI.

Substituting expressions for the constants $c_{1}, c_{3}$ and $c_{5}$ into Equation 3.49 from Pacholczyk (1970) yields an expression for the emission coefficient in CGS units:

$$
\epsilon_{C G S}^{(1),(2)}=\frac{\beta}{16 \sqrt{3}}\left(1 \pm \frac{\gamma+1}{\gamma+7 / 3}\right) \frac{e^{2}}{c}\left(\frac{3 e}{2 \pi m c}\right)^{(1+\gamma) / 2}\left(m c^{2}\right)^{-(\gamma-1)} \mathcal{N}_{0} H_{\perp}^{(1+\gamma) / 2} \nu^{(1-\gamma) / 2}
$$

where, $-e$ is the electron charge, $m$ is the electron mass, $c$ is the speed of light in free space, $H_{\perp}$ is the component of magnetic field intensity perpendicular to the line of sight, and the numerical term $\beta$ is given by

$$
\beta=\Gamma\left(\frac{3 \gamma-1}{12}\right) \Gamma\left(\frac{3 \gamma+7}{12}\right) \frac{\gamma+7 / 3}{\gamma+1}
$$

The plus sign in Equation B1 refers to polarization 1 ( $E$ perpendicular to the magnetic field) and the minus sign to polarization 2 ( $E$ parallel to the field). It is now straightforward to convert this expression to SI, resulting in the formula

$$
\epsilon^{(1),(2)}=\frac{\beta}{16 \sqrt{3}}\left(1 \pm \frac{\gamma+1}{\gamma+7 / 3}\right) \frac{e^{2}}{4 \pi \epsilon_{0} c}\left(\frac{3 e}{2 \pi m}\right)^{(1+\gamma) / 2}\left(m c^{2}\right)^{-(\gamma-1)} \mathcal{N}_{0} B_{\perp}^{(1+\gamma) / 2} \nu^{(1-\gamma) / 2}
$$

where $\epsilon_{0}$ is the permittivity of free space and $B_{\perp}=\left(B_{a}^{2}+B_{b}^{2}\right)^{1 / 2}$. It follows that the constant $C$ in all expression for the emission coefficients is given by

$$
C(\gamma)=\frac{\beta}{16 \sqrt{3}} \frac{e^{2}}{4 \pi \epsilon_{0} c}\left(\frac{3 e}{2 \pi m}\right)^{(1+\gamma) / 2}\left(m c^{2}\right)^{-(\gamma-1)}
$$

Similarly, substituting for $c_{6}$ and $c_{1}$ in Equation 3.51 from Pacholczyk (1970) yields an expression for the absorption coefficients

$$
\kappa_{C G S}^{(1),(2)}=\frac{\alpha}{16 \sqrt{3}}\left(1 \pm \frac{\gamma+2}{\gamma+10 / 3}\right) \frac{e^{2}}{m c}\left(\frac{3 e}{2 \pi m c}\right)^{(\gamma+2) / 2}\left(m c^{2}\right)^{-(\gamma-1)} \mathcal{N}_{0} H_{\perp}^{(2+\gamma) / 2} \nu^{-(\gamma+4) / 2}
$$

where

$$
\alpha=(\gamma+10 / 3) \Gamma\left(\frac{3 \gamma+2}{12}\right) \Gamma\left(\frac{3 \gamma+10}{12}\right)
$$


Again, this is easily converted to SI, giving

$$
\kappa^{(1),(2)}=\frac{\alpha}{16 \sqrt{3}} \frac{e^{2}}{4 \pi \epsilon_{0} m c}\left(1 \pm \frac{\gamma+2}{\gamma+10 / 3}\right)\left(\frac{3 e}{2 \pi m}\right)^{(\gamma+2) / 2}\left(m c^{2}\right)^{-(\gamma-1)} \mathcal{N}_{0} B_{\perp}^{(2+\gamma) / 2} \nu^{-(\gamma+4) / 2}
$$

It follows (by comparison with Equation 9) that the constant $D$ in the expressions for the absorption coefficients is given by

$$
D(\gamma)=\frac{\alpha}{16 \sqrt{3}} \frac{e^{2}}{4 \pi \epsilon_{0} m c}\left(\frac{3 e}{2 \pi m}\right)^{(\gamma+2) / 2}\left(m c^{2}\right)^{1-\gamma}
$$

One further constant of importance appears in the term $\epsilon / \kappa$, which appears when the expressions for the emission and absorption coefficients are substituted into the uniform source solutions, Equations 19 and 20.

$$
\begin{aligned}
\left(\frac{\epsilon}{\kappa}\right)^{(a),(b)} & =\frac{C \mathcal{N}_{0}(K) B_{0}^{(1+\gamma) / 2} \nu^{-(\gamma-1) / 2}}{D \mathcal{N}_{0}(K) B_{0}^{(2+\gamma) / 2} \nu^{-(\gamma+4) / 2}}\left(\frac{F_{\gamma}}{F_{\gamma+1}}\right)^{(a),(b)} \\
& =\mu \frac{m \nu^{5 / 2}}{\nu_{L}^{1 / 2}}\left(\frac{F_{\gamma}}{F_{\gamma+1}}\right)^{(a),(b)}
\end{aligned}
$$

where $\nu_{L}=e B_{0} /(2 \pi m)$ is the cyclotron frequency in magnetic field $B_{0}$ and $\mu$ is the numerical value given by

$$
\mu=\frac{\Gamma\left(\frac{3 \gamma-1}{12}\right) \Gamma\left(\frac{3 \gamma+7}{12}\right)(\gamma+7 / 3)}{\sqrt{3} \Gamma\left(\frac{3 \gamma+2}{12}\right) \Gamma\left(\frac{3 \gamma+10}{12}\right)(\gamma+10 / 3)(\gamma+1)}
$$

Numerical values of $\alpha, \beta$ and $\mu$ are given for common values of $\gamma$ in Table A1.

\section{Variation of $\Pi_{t h i c k}$ with $K$ in a simple model.}

Table 1: Numerical values for $\alpha, \beta$ and $\mu$.

\begin{tabular}{llll}
\hline \hline$\gamma$ & $\beta$ & $\alpha$ & $\mu$ \\
\hline 1.5 & 4.847 & 7.261 & 0.385 \\
2.0 & 2.945 & 6.449 & 0.264 \\
2.5 & 2.074 & 6.063 & 0.198 \\
3.0 & 1.612 & 5.961 & 0.156 \\
3.5 & 1.347 & 6.081 & 0.128 \\
\hline
\end{tabular}


This section presents a magnetic field model that is similar to, but simpler than, that discussed in the main text of this paper. The aim is to illustrate more clearly the origin of the unusual behaviour of $\Pi_{\text {thick }}$ shown in Fig. 3 (lower panel) in which, as $K$ increases from zero, (reducing the anisotropy of the magnetic field) then for $\delta=0, \Pi_{\text {thick }}$ actually increases. This behaviour is more easily understood in the case of a source in which the magnetic field is in the plane of the sky. It consists of a large number of cells, a fraction $(1-x)$ of which cells have magnetic field $B$ parallel to the $b$ axis with value $B_{0} / K$, and a fraction $x$ have $B$ parallel to the $a$ axis with value $B_{0}(K<1)$. The fact that this field configuration doesn't satisfy $\nabla . \mathbf{B}=0$ does not detract from its usefulness for the present purpose.

Since the magnetic field is in the sky plane in one of two orthogonal directions, Equations 8 and 9 give the emission and absorption coefficients for the fraction $(1-x)$ of cells with $B$ parallel to the $b$ axis as

$$
\begin{aligned}
\epsilon^{(a),(b)} & =C N\left(B_{0} / K\right)^{3 / 2}(1 \pm s) \\
\kappa^{(a)(, b)} & =D N\left(B_{0} / K\right)^{2}(1 \pm r)
\end{aligned}
$$

where, for $\gamma=2, s=(\gamma+1) /(\gamma+7 / 3)=9 / 13, r=(\gamma+2) /(\gamma+10 / 3)=3 / 4$ and the upper and lower symbols in the plus or minus sign refer to polarizations $(a)$ and $(b)$ respectively. For the fraction $x$ of cells with magnetic field parallel to the $a$ axis, the $(1+r)$ factors become $(1-r)$ and vice versa, and similarly for $(1 \pm s)$. The magnetic field becomes $B_{0}$. For these cells, the emission and absorption coefficients are therefore

$$
\begin{aligned}
\epsilon^{(a,(b))} & =C N\left(B_{0}\right)^{3 / 2}(1 \mp s) \\
\kappa^{(a, b))} & =D N\left(B_{0}\right)^{2}(1 \mp r)
\end{aligned}
$$

The total contribution to $\epsilon^{(a)}$ is therefore

$$
\begin{aligned}
\epsilon^{(a)} & =C N_{0}\left(B_{0} / K\right)^{3 / 2}\left((1-x)(1+s)+K^{3 / 2} x(1-s)\right) \\
& =C N_{0}\left(B_{0} / K\right)^{3 / 2}(1-x)(1+s)\left(1+\frac{x}{1-x} \frac{1-s}{1+s} K^{3 / 2}\right)
\end{aligned}
$$

Similarly, the remaining coefficients are

$$
\begin{aligned}
\epsilon^{(b)} & =C N_{0}\left(B_{0} / K\right)^{3 / 2}(1-x)(1-s)\left(1+\frac{x}{1-x} \frac{1+s}{1-s} K^{3 / 2}\right) \\
\kappa^{(a)} & =D N_{0}\left(B_{0} / K\right)^{2}(1-x)(1+r)\left(1+\frac{x}{1-x} \frac{1-r}{1+r} K^{2}\right) \\
\kappa^{(b)} & =D N_{0}\left(B_{0} / K\right)^{2}(1-x)(1-r)\left(1+\frac{x}{1-x} \frac{1+r}{1-r} K^{2}\right)
\end{aligned}
$$


The degree of polarization is

$$
\Pi_{\text {thick }}=\frac{\left(\epsilon^{(a)} / \kappa^{(a)}\right)-\left(\epsilon^{(b)} / \kappa^{(b)}\right)}{\left(\epsilon^{(a)} / \kappa^{(a)}\right)+\left(\epsilon^{(b)} / \kappa^{(b)}\right)}=\frac{Q-1}{Q+1}
$$

where $Q=\left(\epsilon^{(a)} \kappa^{(b)}\right) /\left(\epsilon^{(b)} \kappa^{(a)}\right)$. If $K \rightarrow 0, \Pi_{\text {thick }}$ is as for a uniform field, i.e. negative, and $0<Q<1$. Then, if $Q$ increases with increasing $K,\left|\Pi_{\text {thick }}\right|$ decreases. If $Q$ decreases with increasing $K$, then $\left|\Pi_{\text {thick }}\right|$ increases. Substituting from Equations, C5 to C9,

$$
Q=\frac{1+s}{1-s} \frac{1-r}{1+r} \frac{1+X S K^{3 / 2}}{1+X S^{-1} K^{3 / 2}} \frac{1+X R^{-1} K^{2}}{1+X R K^{2}}
$$

where $S=(1-s) /(1+s), R=(1-r) /(1+r)$, and $X=x /(1-x)$. If $K \ll 1$, then, neglecting terms of order $K^{3}$ and higher

$$
\begin{aligned}
Q & =\frac{R}{S}\left(1+X\left(S-S^{-1}\right) K^{3 / 2}+X\left(R^{-1}-R\right) K^{2}\right) \\
& =\frac{R}{S}\left(1+X\left(\frac{-4 s K^{3 / 2}}{1-s^{2}}+\frac{4 r K^{2}}{1-r^{2}}\right)\right)
\end{aligned}
$$

which will decrease with increasing $K$ if $d Q / d K<0$, i.e. if

$$
\frac{3 s K^{1 / 2}}{2\left(1-s^{2}\right)}>\frac{2 r K}{1-r^{2}}
$$

or

$$
K<\frac{9}{16}\left(\frac{s}{1-s^{2}} \frac{1-r^{2}}{r}\right)^{2}=0.34
$$

to two significant figures, if $\gamma=2$. So provided $K$ is very small, as $K$ increases, $Q$ decreases and $\left|\Pi_{\text {thick }}\right|$ increases, while $\Pi_{\text {thick }}$ is negative. This happens because as $K$ increases, the emission process tends to favour $I^{(b)}$ over $I^{(a)}$ (i.e. $\epsilon^{(b)}$ increases more than $\epsilon^{(a)}$ ). However, the absorption process (or the mean free path) favours $I^{(b)}$ over $I^{(a)}$ (because $\kappa^{(b)}$ increases more than $\kappa^{(a)}$ ). If $K$ is small, the contribution of the weak field cells to emission dominates their contribution to absorption, because they contribute a greater fraction toward the total emission coefficient $\left(\propto K^{3 / 2}\right)$ than toward the total absorption coefficient $\left(\propto K^{2}\right)$.

Comparison with Inequality $\mathrm{C} 13$ with the position of the turning point on the $\delta=0$ curve from the lower panel of Fig. 3, shows that, in the compressed random field model, $\left|\Pi_{\text {thick }}\right|$ increases with $K$ over a more limited range of $K$, from $K=0$ to about 0.2 , rather than 0.34. This discrepancy arises because, in the compressed random field model, when $K$ is small, the field orthogonal to the $a$ axis has structure similar to a plate of spaghetti: much of it points toward the observer, reducing the emission coefficients of this component by $\sin ^{3 / 2} \theta$ and the absorption coefficients by $\sin ^{2} \theta$, where $\theta$ is the inclination of the field to the line of sight. The result is to replace $K$ in the above expressions by $K / \sin \theta$. This will tend to make the condition on $K$ more stringent than given by Inequality C13. 


\section{REFERENCES}

Agudo, I., Gómez, J. L., Casadio, C., Cawthorne, T. V., \& Roca-Sogorb, M. 2012, ApJ, 752, 92

Cawthorne, T. V. 2006, MNRAS, 367, 851

Hughes, P. A., Aller, H. D., \& Aller, M. F. 1985, ApJ, 298, 301

Hughes, P. A., Aller, H. D., \& Aller, M. F. 1989, ApJ, 341, 54

Hughes, P. A., Aller, H. D., \& Aller, M. F. 1989, ApJ, 341, 68

Hughes, P. A., Aller, H. D., \& Aller, M. F. 1991, ApJ, 374, 57

Hughes, P. A., Aller, M. F., \& Aller, H. D. 2011, ApJ, 735, 81

Jones, T. W., Rudnick, L., Aller, H. D., et al. 1985, ApJ, 290, 627

Laing, R. A. 1980, A model for the magnetic-field structure in extended remacsadio sources, MNRAS, 193, 439

Laing, R. A., \& Bridle, A. H. 2002, MNRAS, 336, 328

Laing, R. A., Canvin, J. R., Bridle, A. H., \& Hardcastle, M. J. 2006, MNRAS, 372, 510

Marscher, A. P. 2006, Relativistic Jets: The Common Physics of AGN, Microquasars, and Gamma-Ray Bursts, 856, 1

Marscher, A. P., Jorstad, S. G., Larionov, V. M., et al. 2010, ApJ, 710, L126

McKinney, J. C., Tchekhovskoy, A., \& Blandford, R. D. 2012, MNRAS, 423, 308

Pacholczyk, A. G. 1970, Radio astrophysics, San Francisco: Freeman, 1970

Porth, O. 2013, MNRAS, 429, 2482

Pudritz, R. E., Hardcastle, M. J., \& Gabuzda, D. C. 2012, Space Sci. Rev., 169, 27

Wehrle, A. E., Marscher, A. P., Jorstad, S. G., et al. 2012, ApJ, 758, 72

Wilson, A. S., Young, A. J., \& Shopbell, P. L. 2001, ApJ, 547, 740 